\title{
Rheological parameters identification using in situ experimental data of a flat die extrusion
}

\author{
N. Lebaal ${ }^{\mathrm{a}, *}$, S. Puissant ${ }^{\mathrm{a}}$, F.M. Schmidt ${ }^{\mathrm{b}}$ \\ a Institut Supérieur d'Ingénierie de la Conception, Equipe de Recherche en Mécanique et Plasturgie, \\ 27 rue d'Hellieule, 88100 Saint-Dié-des-Vosges, France \\ ${ }^{\mathrm{b}}$ Ecole des mines d'Albi Carmaux, Laboratoire CROMeP, Campus Jarlard-Route de Teillet, 81013 Albi Cedex 9, France
}

\begin{abstract}
Viscosity is an important characteristic of flow property's and process ability for polymeric materials. A flat die was developed by Mailleferextrusion, to make rheological characterisations. In this paper, the rheological parameters of the melt are identified through optimisation by a response surface method. The objective is to minimize the differences between the measured pressure obtained in flat die and the pressures computed by one-dimensional finite difference code programme. An objective function is defined as the global relative error obtained through the differences between measured and computed pressures. This objective function is minimised by varying the rheological parameters. For this minimisation, two methods are used, i.e. the local response surface and the global response surface. The rheological parameters permit to calculate the viscosity. Then, we compare this calculated viscosity with an experimental viscosity measured on a capillary rheometer to validate our method.
\end{abstract}

Keywords: Polymer; Extrusion; Numerical simulation; Identification

\section{Introduction}

The defects of extrusion (like the weld-lines, the fairly uniform exit velocity distribution throughout the extrusion and problems of stagnation zones) are influenced by the geometry of the die of extrusion as well as by the operating conditions such as temperature of regulation, flow rate and the rheological parameters of melt. In fact, if the rheology of most generic polymers is well known, the plastics used on production extrusion lines are seldom known because the polymer is obtained by blending or adding different additives. So, in the first step and in order to validate the method of optimisation, we will identify the rheological (behaviour) parameters of the plastic melts starting from the experimental data obtained in a flat die. Our objective is to identify the rheology of a plastic melt directly from on-line production, without using conventional measurements on dies capillary standard.

A certain number of viscosity models have been published $[1,2]$, such as the well-known Power-law model, the

\footnotetext{
* Corresponding author.

E-mail address: lebaal@enstimac.fr (N. Lebaal).
}

Ostwald-Waele model, the Cross model, the Carreau model, and so on. Liang [3] investigated the melt viscosity in steady shear flow of several polymers by employing a capillary rheometer and the characteristics of shear viscosity. Geiger and Kuhnle [4] used a empirical correlation between density, pressure and temperature to obtain a rheological model. Choosing one of these rheological laws, we will identify its parameters. We will use classical design of experiments [7] and algorithm of optimisation called the response surface method, with a moving least squares approximation $[5,6]$. The experimental points are obtained with a standard extruder feeding at various flow rates of the flat die. Two series of tests were carried out, with different thickness of die to cover a sufficient range of shear rate to make rheological characterisations.

\section{The optimisation benchmark}

A flat die produced by Maillefer-extrusion [8] (Fig. 1) is equipped with four pressure transducers, spaced $100 \mathrm{~mm}$ apart; however, only the pressure difference between the first 


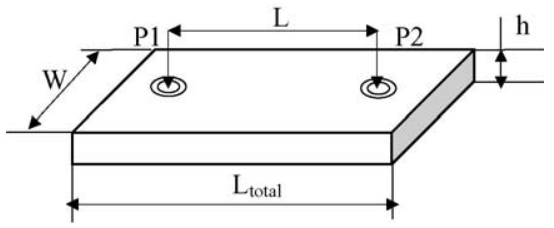

Fig. 1. Geometry of the flat die.

Table 1

Experimental data slit 5 and $10 \mathrm{~mm}$

\begin{tabular}{clclc}
\hline$Q(\mathrm{~kg} / \mathrm{h})$ & $T(\mathrm{~K})$ & $\Delta P(\mathrm{bar})$ & $\rho\left(\mathrm{kg} / \mathrm{m}^{3}\right)$ & $h(\mathrm{~mm})$ \\
\hline 11.2 & 439 & 53 & 779 & 5 \\
60 & 448 & 99 & 779 & 5 \\
143 & 465 & 125 & 773 & 5 \\
239 & 483 & 138 & 767 & 5 \\
10.9 & 458 & 12.7 & 779 & 10 \\
69.2 & 468 & 26 & 772 & 10 \\
189.5 & 483 & 34.3 & 761 & 10 \\
339.5 & 502 & 36.7 & 755 & 10 \\
\hline
\end{tabular}

and the last transducer was considered for the analysis. A first series of tests has been realized with the flat die. Measurements were made with two different slit heights at different extrusion speeds for one type of material (LDPE). The experimental values are listed in Table 1 . The properties of the polymer (a LDPE grade) are referenced in Table 2.

\section{Numerical simulation parameters}

The measurement of viscosity cannot be obtained directly because the shear rate is unknown as well as the three parameters for the Power-law. On the other hand, three physical parameters related to viscosity such as pressure, temperature and the flow rate are directly measurable. We will use onedimensional finite difference calculation software by sections $[1,2]$, to calculate the variation of pressure with the Powerlaw for each point of measurement. The choice of this geometry was made in order to respect correction factor $[9,10]$. The shape factor, which is the thickness to width ratio, $h / W$ equals 0.1 and 0.05 .

\section{Design variables and objective functions}

The Power-law is defined as:

$\eta=K_{0} \mathrm{e}^{\beta\left(\frac{1}{T}\right)} \dot{\bar{\gamma}}^{m-1}$

Table 2

Properties of the polymer under study and geometry

\begin{tabular}{lc}
\hline Specific heat, $C_{\mathrm{p}}(\mathrm{J} /(\mathrm{kg} \mathrm{K}))$ & 1900 \\
Conductivity, $k\left(\mathrm{~W} /\left({ }^{\circ} \mathrm{C} \mathrm{m}\right)\right)$ & 0.115 \\
$L_{\text {total }}(\mathrm{mm})$ & 500 \\
$L(\mathrm{~mm})$ & 300 \\
$W(\mathrm{~mm})$ & 100 \\
\hline
\end{tabular}

There are three variables $(K, m$ and $\beta$ ) for a Power-law with a thermal dependence of the Arrhenius type.

The objective of this simulation to identify the rheological parameters $K, m$ and $\beta$. This is done by minimizing the objective function representing the value of the sum of the quadratic difference between the calculated pressures and the experimental pressures for all point of measurement.

$J=\left(\frac{\sum_{\mathrm{npm}}\left(p_{\exp }-p_{\mathrm{cal}}\right)}{p_{\exp }}\right)^{2}$

\section{Optimisation procedure}

\subsection{Choice of algorithm}

The algorithm of optimisation must be carefully chosen when one single analysis requires several hours of CPU time. Non-deterministic or stochastic methods such as MonteCarlo method and genetic algorithm [11] can obtain global minimum, but they need a lot of evaluations for the functions to converge. Gradient methods [12] require the computations of the gradients of the functions, such as BFGS [13] and SQP [14]. The computation of gradients by finite difference is time consuming and depends on the perturbed parameters. For the above reasons, we decided to choice a response surface method.

\subsection{Response surface method}

The method of response surface consists of the construction of an approximate expression of objective function starting from a limited number of evaluations of the real function.

The main idea is to approximate the objective function through a response surface. In order to obtain a good approximation, we used a moving least square method. In this method, the approximation is computed by using the evaluation points by design of experiments around the locus, where the value of the function is needed.

\section{Moving least-squares approximation}

We will use moving least-square interpolations in order to approximate the response surface of objective function. Let the dependent variable be $f(x)$ in the domain $S$ and the approximation $\tilde{f}(x)$.

$\tilde{f}(x)=p(x) a^{\mathrm{T}}(x)$

The nodes are defined by $x_{1}, \ldots, x_{n}$, where $x_{1}=\left(x_{1}, y_{1}\right)$ in two-dimensional (2D), $x_{1}=\left(x_{1}, y_{1}, z_{1}\right)$ in three-dimensional (3D); $p(x)$ is a polynomial basis and $a^{\mathrm{T}}(x)$ is the vector of coefficients. The polynomial basis of order two in two di- 


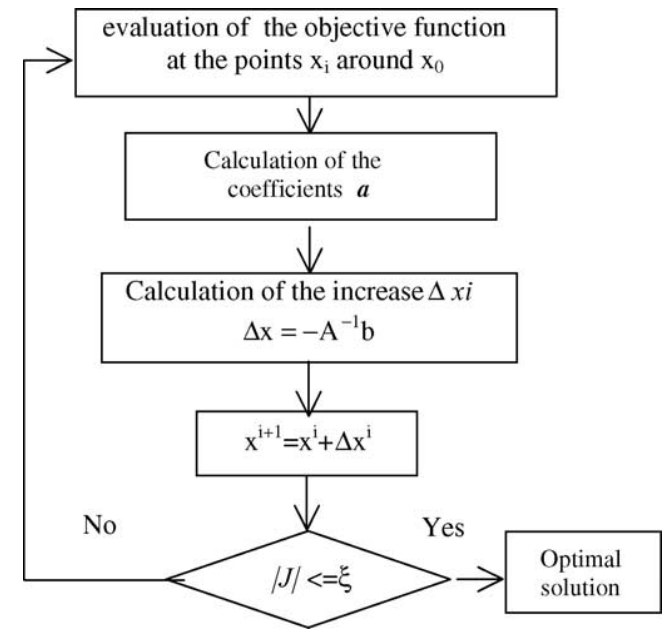

Fig. 2. Optimisation algorithm.

mensions is given by:

$P_{i}=\left[1(x)(y) \frac{1}{2}(x)^{2}(x)(y) \frac{1}{2}(y)^{2}\right]$

In the moving least-square interpolation, at each point $\mathrm{x}$, $\mathrm{a}$ is chosen to minimize the weighted residual:

$$
\begin{aligned}
J(a) & =\sum w_{i}\left(f_{i}-P(x)^{\mathrm{T}} a(x)\right)^{2} \\
& \Rightarrow J(a)=\frac{1}{2}(F-\mathrm{Pa})^{\mathrm{T}}(F-\mathrm{Pa})
\end{aligned}
$$

where $w_{i}$ is a weight function, such that $w$ is non-zero over a domain of influence.

In order to find the coefficients, we obtain the minimum of $J(a)$ by

$J^{\prime}(a)=0$

So we have:

$a(x)=Q^{-1} B Z$

where

$Q=P^{\mathrm{T}} W P$

and

$B=P^{\mathrm{T}} W Q$

The iterative procedure stops when the successive points are superposed with a certain tolerance $\varepsilon=10^{-3}$.

\section{Local response surface}

This method is based on traditional minimisation by the algorithm of Fig. 2, with the use of a local response surface of variable in each point. We fixed a step of grid at $0.01 \times X_{0}$, where $X_{0}$ is the initial value of the variable of optimisation.

The initial values are: $K_{0}=12,000 \mathrm{~Pa} \mathrm{~s}, m_{0}=0.64$ and $\beta_{0}=1078 \mathrm{~K}$. We used a uniform local grid.
The constraints for the variables of optimisation $K, m$ and $\beta$ are, respectively, $400 \mathrm{Pas}<K<20,000 \mathrm{~Pa} \mathrm{~s}, 0.01<m<1$ and $500 \mathrm{~K}<\beta<3000 \mathrm{~K}$ for the first constraint, and $n \times P$ for the second constraint, where $P$ is the step of the grid and $n$ is the number of points of grid.

\section{Global response surface}

The objective of this technique is to reach the global minimum on the field domain: the constraints are similar to the previous constraints in the local response surface method. Initially, at first iteration we constructed a grid of $5 \times 5 \times 5$ points ( 125 points) for a global approximation. Then, for each iteration, we constructed a grid of $3 \times 3 \times 3$ (27 points) for the local approximation.

\section{Results}

\subsection{Local response surface}

Fig. 3 represents the evolution of the objective function and Figs. 4-6 represent, respectively, the variation of the coefficients of the constitutive law $K, m$ and $\beta$ during the process of optimisation. In iteration 20 , the value of the function objective obtained is $8 \times 10^{-5}$, with $K=9826 \mathrm{~Pa} \mathrm{~s}, m=0.4179$

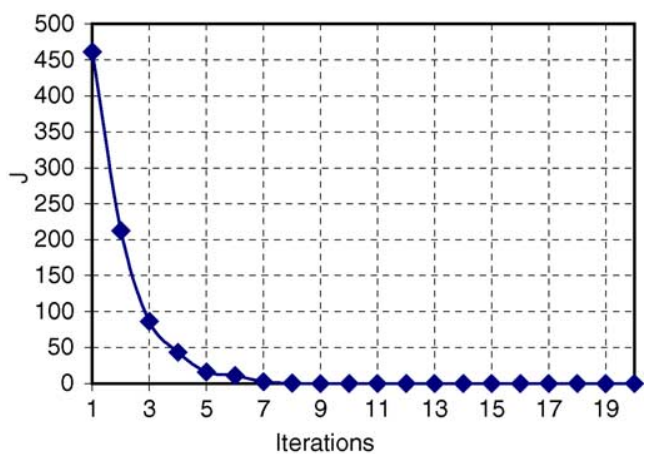

Fig. 3. Convergence of the objective function (the local method).

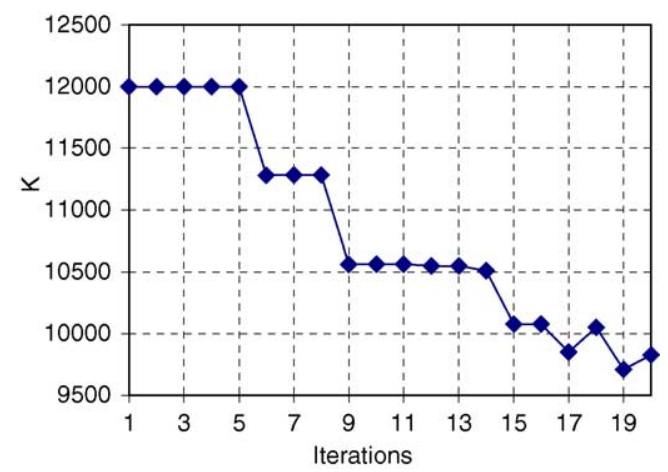

Fig. 4. Evolution of $K$ during the process of optimisation (the local method). 


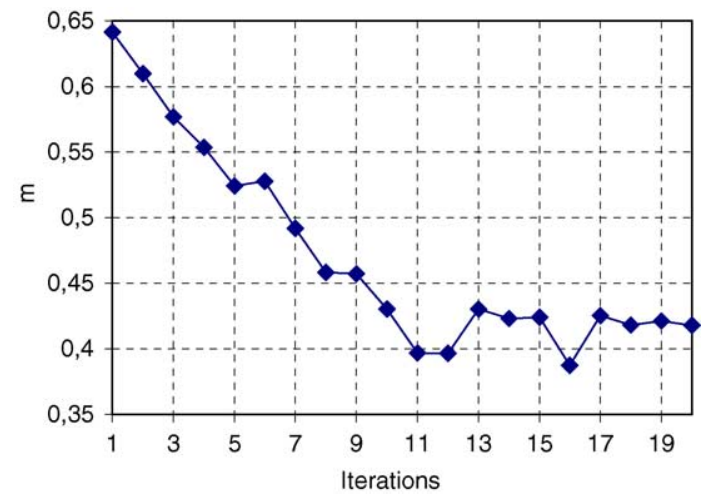

Fig. 5. Evolution of $m$ during the process of optimisation (the local method).

and $\beta=1553 \mathrm{~K}$. The CPU time is $1 \mathrm{~h}$ and $42 \mathrm{~min}$ on a computer Pentium IV, $2.4 \mathrm{GHz}, 512$ Mo RAM.

We can choose between precision and CPU time: for example, in iteration 10 , we obtained an error of $6.848 \times 10^{-3}$ with a time CPU $51 \mathrm{~min}$; the values of the rheological parameters are: $K=10,562 \mathrm{~Pa} \mathrm{~s}, m=0.4304, \beta=1131 \mathrm{~K}$. With iteration 15 , we obtained an error of $5.2 \times 10^{-4}$, with a computing time of $1 \mathrm{~h}$ and $16 \mathrm{~min}$ in the same machine, the coefficients obtained are: $K=10,077 \mathrm{~Pa} \mathrm{~s}, m=0.4239$ and $\beta=1350 \mathrm{~K}$.

In Fig. 4, the evolution of the parameter $K$ is shown in function of the iteration. We can see that it is varying quite fast at the beginning of the optimisation, until the ninth step. Its total variation is about $16 \%$.

In Fig. 5, the evolution of the parameter $m$ in function of the iteration, we notice that the variation is fast at the beginning of the optimisation, until the 11th step. Its total variation (36\%) is more important than for $K$.

As in Fig. 7, the variation of beta in function of iteration step, we can observe that this parameter is quite stable at the beginning and starts to change after iteration 11, when $K$ and $m$ have almost their final value.

\subsection{Result of global response surface}

The solution is obtained after six iterations with a precision of $8.4 \times 10^{-4}$ and a CPU time is $1 \mathrm{~h}$ and $13 \mathrm{~min}$. Fig. 7 rep-

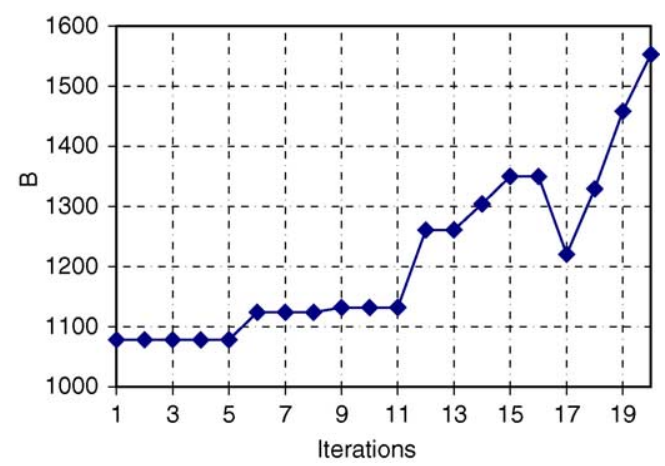

Fig. 6. Evolution of $\beta$ during the process of optimisation (the local method).

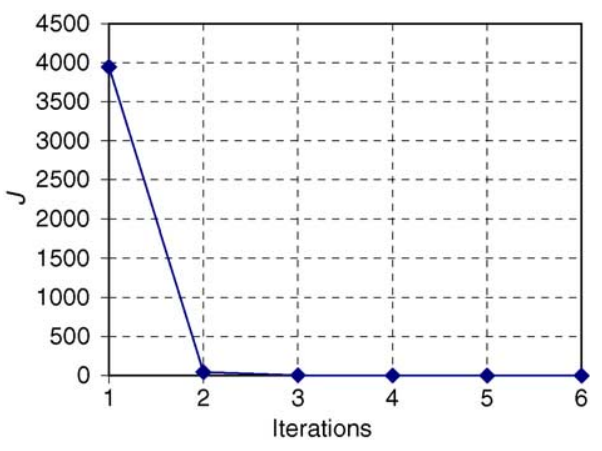

Fig. 7. Convergence of the objective function (the global method).

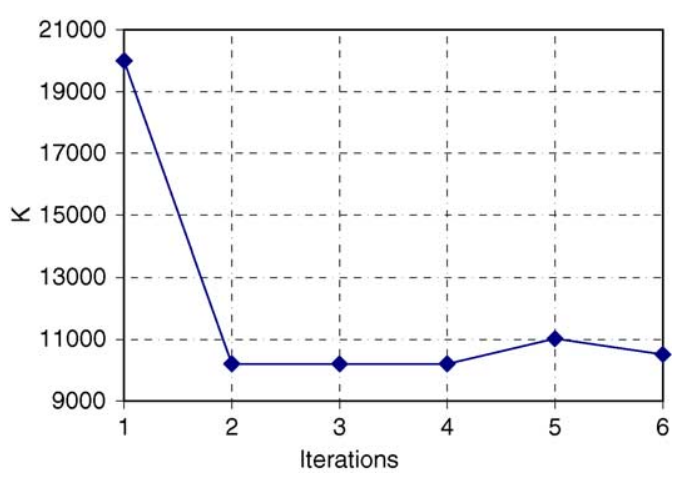

Fig. 8. Evolution of $K$ during the process of optimisation (the global method).

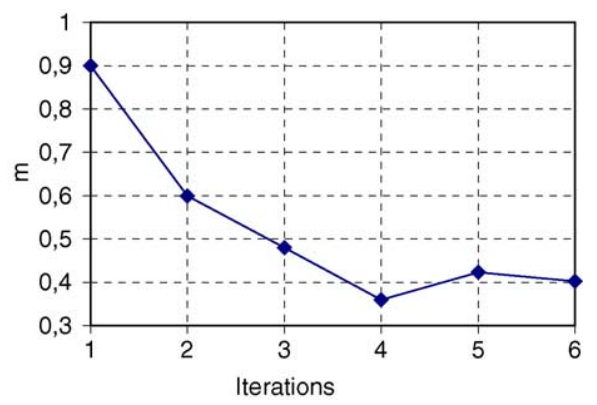

Fig. 9. Evolution of $m$ during the process of optimisation (the global method).

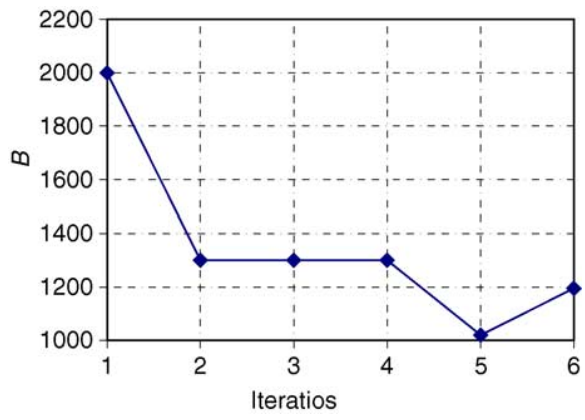

Fig. 10. Evolution of $\beta$ during the process of optimisation (the global method). 
Table 3

Results of optimisation

\begin{tabular}{|c|c|c|c|c|c|}
\hline$h(\mathrm{~mm})$ & $\Delta P$ measured (bar) & $\Delta P$ (bar) local (1) recalculated & Relative error (1) & $\Delta P$ (bar) global (2) recalculated & Relative error (2) \\
\hline 5 & 53 & 52.11 & $2.8 \times 10^{-4}$ & 49.32 & $4.8 \times 10^{-3}$ \\
\hline 5 & 99 & 96.69 & $5.4 \times 10^{-4}$ & 91.03 & $6.4 \times 10^{-3}$ \\
\hline 5 & 125 & 121.6 & $7.3 \times 10^{-4}$ & 116.8 & $4.3 \times 10^{-3}$ \\
\hline 5 & 138 & 132.9 & $1.3 \times 10^{-3}$ & 130.4 & $3.0 \times 10^{-3}$ \\
\hline 10 & 12.7 & 12.49 & $2.7 \times 10^{-4}$ & 12.48 & $3.0 \times 10^{-4}$ \\
\hline 10 & 26 & 25.15 & $1.0 \times 10^{-3}$ & 24.89 & $1.8 \times 10^{-3}$ \\
\hline 10 & 34 & 34.69 & $1.2 \times 10^{-4}$ & 34.66 & $3.7 \times 10^{-4}$ \\
\hline
\end{tabular}

resents the evolution of the function objective and Figs. 8-10 represent, respectively, the variables of optimisation $K, m$ and $\beta$. The optimum parameters are: $K=10,500 \mathrm{~Pa} \mathrm{~s}, m=0.4027$ and $\beta=1194 \mathrm{~K}$.

We can observe that the convergence process is much faster in terms of iteration steps needed. But the computation time is longer. Also, in this method, the three parameters are evolving together in the first iteration steps before reaching values close to the final one.

\section{Comparison of the two methods}

In Table 3, in function of the operating parameters (slit height, output and temperature), we have reported the pressures measured and recalculated using the identified rheological parameters, so as the relative error (relative difference between the two). We notice that the solution obtained by the method (1) local response surfaces is more accurate, but with an increased computing time. The difference in time between both methods is $30 \mathrm{~min}$. On the other hand, the global response surface method (2) avoids local minimum. This can be improved by varying the design of experiments and the method of approximation to optimise the computing time CPU.

\subsection{Pressures}

In Fig. 11, the measured pressures and computed pressures obtained with the identified rheological parameters are

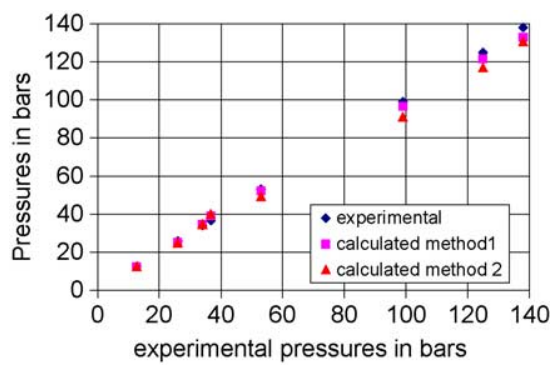

Fig. 11. Experimental and calculated pressure with parameters determinate by method (2).

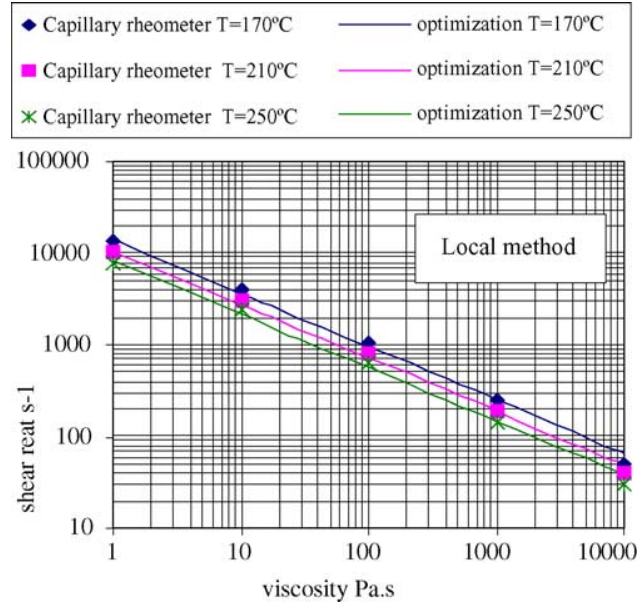

Fig. 12. Computed and measured viscosity method (1).

plotted. There is only a small discrepancy, which means that our method is very robust and gives very good results for both global and local methods. This is also confirmed through the relative error for methods (1) and (2), which are, respectively, $9.14 \times 10^{-3}$ and $2.9 \times 10^{-2}$.

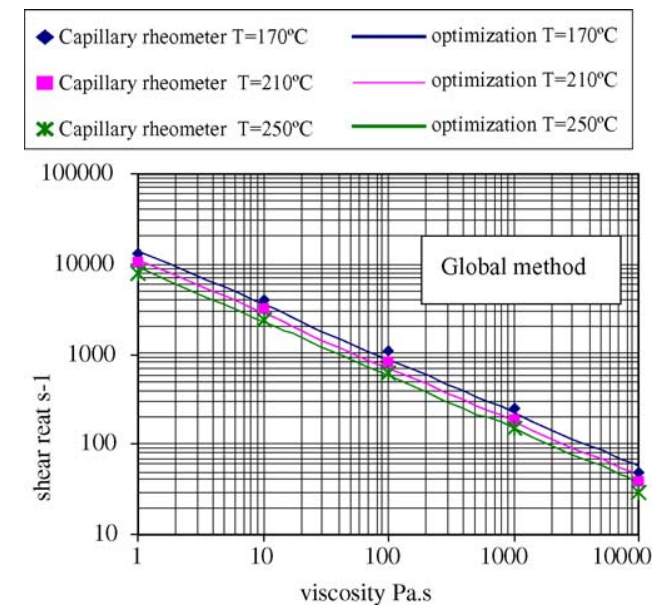

Fig. 13. Computed and measured viscosity method (2). 


\subsection{Viscosity}

In the graph of viscosity obtained by the coefficients found through the response surface method (Figs. 12 and 13), we can observe that there is no discrepancy between the points of measurements obtained in capillary rheometer and the viscosity obtained by the method of optimisation except in the zone, where high shear rates appear.

\section{Conclusion and prospects}

We conclude that we obtain good results using the response surface methods (global and local). It means that this method allows us to determine the rheological parameters of polymers directly on a production line. By on will not forced to make measurements on standard capillary rheometers. And also this method will allow us to measure the parameters in industrial conditions, starting from the real geometry even if complex, using pressure measurements, flow rates and temperatures.

\section{Future works}

Also to test the Kriging method [15], we will have to improve precision (CPU time) of the global response surface approximation.

We will use the same approximation method to apply on a real 3D geometry to identify the rheological parameters and then to optimise the geometry of die extrusion tolls. The extrusion simulations will be processed by using the REM3D ${ }^{\circledR}$ [16] FEM software.

\section{Acknowledgment}

The support of Maillefer-extrusion is gratefully acknowledged.

\section{References}

[1] J.F. Agassant, P. Avenas, J.P.H. Sergent, P.J. Carreaur, Polymer Processing. Principles and Modelling, Hanser Publishers, 1991.

[2] C. Rauwendahl, Polymer Extrusion, 3rd ed., Hanser Publishers, 1994.

[3] J.-Z. Liang, Characteristics of melt shear viscosity during extrusion of polymers, Polym. Test. 21 (2002) 307-311.

[4] K. Geiger, H. Kuhnle, Analytische Berechnung einfacher scherstromungen aufgrund eines fliebgesetzes vom carreauschen typ, Rheogica Acta 23 (1984) 355-367.

[5] K.M. Liew, Y.Q. Huang, J.N. Reddy, Vibration analysis of symmetrically laminated plates based on FSDT using the moving least squares differential quadrature method, Comput. Methods Appl. Mech. Eng. 192 (2003) 2203-2222.

[6] Y. Krongauz, T. Belytschko, Enforcement of essential boundary conditioned in mesh less approximations using finite elements, Comput. Methods Appl. Mech. Eng. 131 (1996) 133145.

[7] J. Goupy, Plans d'Expériences Pour Surfaces de Réponse, Industries Techniques, Paris, 1999.

[8] D. Schläfli, Filière Rhéométriques Théorie/Filière Plate, Internal Report of Nokia Maillefer, 1997.

[9] M.A. Huneault, P.G. Lafleur, P.J. Carreau, Evaluation of the FAN Technique for Profile Die Design, International Polymer Processing, vol. XI, 1996, pp. 50-57.

[10] S.J. Kim, T.H. Kwon, A simple approach to determining threedimensional screw characteristics in the metering zone of extrusion processes using a total shape factor, Polym. Eng. Sci. 35 (3) (1995) 274-283.

[11] J.M. Renders, Algorithmes Génétiques et Réseaux de Neurones, Hermès, Paris, 1995.

[12] W. Sun, J. Han, J. Sun, Global convergence of no monotone descent methods for unconstrained optimisation problems, J. Comput. Appl. Math. 146 (2002) 89-98.

[13] J.L. Morales, A numerical study of limited memory BFGS methods, Appl. Math. Lett. (2002) 481-487.

[14] B. Horowitz, S.M.B. Afonso, Quadratic programming solver for structural optimisation using SQP algorithm, Adv. Eng. Software (2002) 669-674.

[15] F. Trochu, P. Terriault, Non-linear modelling of hysteretic material laws by dual Kriging and application, Comput. Methods Appl. Mech. Eng. 151 (1998) 545-558.

[16] REM3D, User's Manuals, Version 2-2, Transvalor, 2004. 